\title{
ELECTROCHEMICAL TEST SYSTEM FOR ANALYSIS OF SULFUR-CONTAINING ORGANIC SUBSTANCES
}

\author{
V.P. Lukovtsev
}

el-02@mail.ru

\section{N.V. Lukovtseva}

\section{V.A. Semenova}

\section{A.N. Frumkin Institute of Physical Chemistry and Electrochemistry, Russian Academy of Sciences, Moscow, Russian Federation}

\begin{abstract}
The paper discusses selection of a set of metals for an electrochemical test system used for the detection of sulfur-containing organic substances. Analytical studies of organic sulfur compounds are very important since these compounds are widely used in medicine, biology, oil refining industry, etc. Quantitative determination and identification of sulfur-containing substances is essential for solving environmental problems. The electrochemical test system for analysis of organic substances is a solution containing a set of cations of various metals that have the ability to form complex compounds with analytes. Thereby, a single electrode can be used to carry out electrochemical multisensory analysis because metal cations play the role of sensors. Thus, there is no need to use a set of several working (indicator) electrodes, the number of which determines the number of informative parameters. Since sets of metals for test systems may slightly differ from each other, we need to select an optimal set for a specific problem. According to the study results, a set of metals for sulfur-containing organic substances consists of zinc, gallium and copper. The test system composed of these metals gives reliable results of electroanalytical determination of organic compounds of this class. The expediency of using such a test system is confirmed by comparative estimation of the Euclidean distances between the vector representations of the analytes and sulfur-containing organic compounds presented in the previously created database. Software for experimental data processing, visualization, and comparative analysis of the vector representations and the database components is developed
\end{abstract}

\section{Keywords}

Inversion voltammetry, sulfurcontaining organic substances, identification, multisensors, electrochemical test system
Received 14.08.2018

Author(s), 2019 
Introduction. Analytical studies of organic sulfur compounds are of great practical interest due to the fact that these compounds are widely used in various fields: medicine, biology, the refining industry, etc. The quantitative determination and identification of sulfur-containing substances is also an important part of the complex tasks related to environmental protection. Currently used methods for identification and comparative analysis of organic compounds based on chemical analysis, thin-layer chromatography and mass spectrometry have several disadvantages, for example, a high percentage of false-positive reactions, equipment cumbersome, low mobility, large mass of the test sample, complexity stages of sample preparation, etc.

In this regard, it is very promising to use electrochemical methods of analysis, which are devoid of most of these shortcomings. The most informative among them is the inverse voltammetry method. However, it is usually used for the analysis of inorganic substances, in particular heavy metals. In this case, careful sample preparation is required, which consists in removing organic substances from the analyzed sample, which significantly affect the type of analytical voltamperogram. It is this ability to change the behavior of an electrochemical system containing cations of various metals that can be used to analyze organic compounds. One of the promising ways to increase the reliability of the results obtained is the use of electrochemical multisensory analysis, which models the principle currently defined by the term "electronic tongue". To implement this method, an approach is proposed [1-6], consisting in the use of a multisensory test system in the form of a solution containing a set of ions of various metals, which have the ability to form complex compounds with organic substances. Thus, the role of a multitude of sensors is performed by the metal cations contained in the developed test system. There is no need to use a set of several working (indicator) electrodes, the number of which should determine the number of informative parameters. The required set of parameters is obtained on a single electrode in the process of removing the inversion voltamperogram. The method of identification of organic substances is based on the results of studies showing that each of them has a different effect on the electrochemical behavior of the multisensor test system.

Electrochemical test system described in the works [1,2], was focused on the analysis of alkaloids. It is obvious that the sets of metals that make up the test systems for the detection and identification of organic compounds belonging to other classes may differ somewhat from each other.

The purpose of the work is to search for a set of metals, on the basis of which a test system is created for analytical studies of sulfur-containing organic substances. 
Experimental methods. Experimental studies were carried out in an electrochemical cell using planar electrodes, providing work on a three-electrode circuit. Such electrodes are a structure in which a working (indicator) and auxiliary electrodes, as well as a reference electrode, are formed on the same plane (polyester film substrate). The working and auxiliary electrodes are made of carbon paste, and the reference electrode is made of paste $\mathrm{Ag} / \mathrm{AgCl}$. The background electrolyte into which cations of various metals were introduced for carrying out the appropriate tests was a solution $\mathrm{KCl}$ by concentration $0.05 \mathrm{M}$.

To set the required modes and measure the electrical parameters of the experiments used, a multifunctional research instrument was used EL-02 [7].

Experiments were performed in the mode of inversion voltammetry with accumulation. Accumulation potential $-1.55 \mathrm{~V}$ (relative to the silver chloride reference electrode), the accumulation time $60 \mathrm{sec}$, potential sweep rate was $1 \mathrm{~V} / \mathrm{sec}$ in the anode side to the value $0 \mathrm{~V}$. Studies were performed in two stages. At the first stage, a voltamperogram was obtained in a solution containing test metal cations without introducing the analyzed organic substance. At the second stage, a voltamperogram was obtained after introducing organic matter into the solution. Quantitative assessment of the result of the experiment was the value of the change in the rate of dissolution of the testing metals before and after the introduction of the analytes into the solution.

Results and its discussion. In the work, the objects of analytical studies were sulfur-containing organic substances: sulfanilamide; thiobarbituric acid; thiourea; unithiol; sulfosalicylic acid; thiamine. Their influence on the rate of electrodissolution of metals such as zinc, gallium, cadmium, lead, copper, cobalt was considered. This effect can be quantified by the change in the peak value of the anodic dissolution current of each metal. Thus, it is possible to choose the metals that are most sensitive to the presence in the solution of a sulfur-containing organic substance. In turn, such an assessment should be the basis for creating an electrochemical test system that provides the necessary analytical studies.

To conduct experimental studies, nitrates of test metals were injected into the background electrolyte with a concentration of $5 \cdot 10^{5} \mathrm{M}$. The concentration of the studied organic substances $1 \cdot 10^{-1} \mathrm{mg} / \mathrm{mL}$.

An example of inversion voltamperogram showing the effect of thiourea on the peak current of anodic dissolution of zinc is shown in Fig. 1.

Similar experiments were carried out with other sulfur-containing organic substances in order to assess their effect on the rate of dissolution of the above test metals. The results of this assessment are shown in the table. They represent the percentage deviation of the peak values of the currents of anodic 


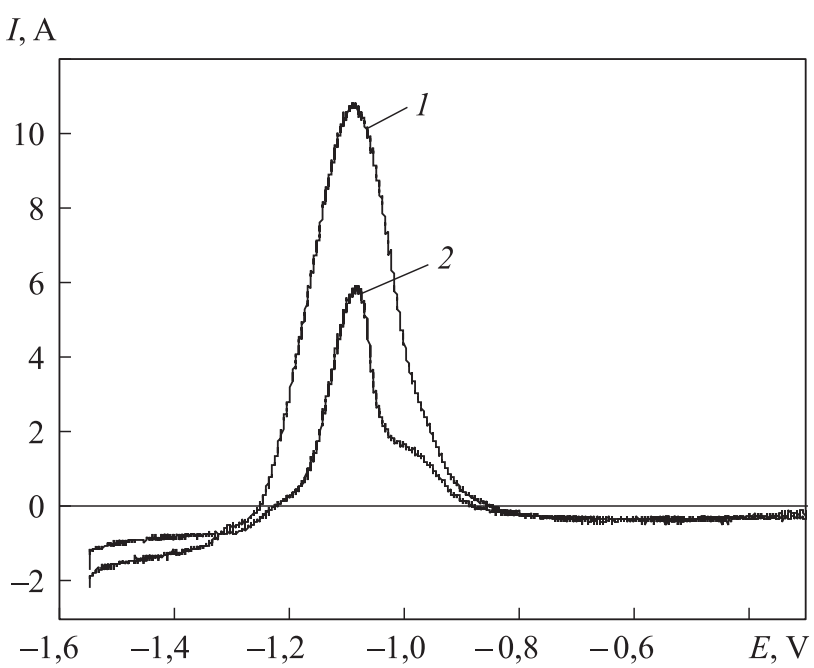

Fig. 1. Inversion voltamperogram for electrolyte containing zinc cations (1) and electrolyte containing zinc cations, with the introduction of thiourea (2)

dissolution of test metals after the injection of the analyzed organic matter into the electrolyte. The table takes into account the fact that on the inversion voltamperogram for electrolytes containing gallium cations, there are two peaks.

The effect of sulfur-containing organic substances on the value of the peaks of the currents of anodic dissolution of test metals (\%)

\begin{tabular}{|c|c|c|c|c|c|c|c|}
\hline \multirow{3}{*}{ Organic matter } & \multicolumn{7}{|c|}{ Metal } \\
\hline & \multirow{2}{*}{$\mathrm{Zn}$} & \multicolumn{2}{|c|}{$\mathrm{Ga}$} & \multirow{2}{*}{$\mathrm{Cd}$} & \multirow{2}{*}{$\mathrm{Pb}$} & \multirow{2}{*}{$\mathrm{Cu}$} & \multirow{2}{*}{ Co } \\
\hline & & Peak 1 & Peak 2 & & & & \\
\hline Sulfanilamide & $-8,3$ & -70 & -60 & $-5,0$ & $+4,7$ & -38 & -12 \\
\hline Thiobarbituric acid & $-2,1$ & -18 & -25 & 0 & -10 & +19 & +28 \\
\hline Silphourea & -45 & +67 & -18 & -12 & $-2,0$ & -66 & -74 \\
\hline Thiourea & $+9,3$ & -21 & 0 & 0 & 0 & -74 & -51 \\
\hline Sulfosalicylic acid & -21 & +20 & +50 & 0 & -12 & -52 & -91 \\
\hline Thiamin & -60 & -100 & -100 & $-1,2$ & 0 & -40 & -50 \\
\hline
\end{tabular}

The presented results of experimental studies allowed to offer a set of metals that make up the electrochemical test system for the analysis of sulfurcontaining organic substances. In drawing up such a kit, it was taken into account that the effect of organic substances on the testing metals should be sufficiently large and, if possible, different for each analyze, and also that the potentials of the peaks of the anodic dissolution of the metals of the test 
V.P. Lukovtsev, N.V. Lukovtseva, V.A. Semenova

system should differ from each other. On the one hand, the number of metals that make up the test system should be sufficient for the implementation of multisensory analysis, on the other hand, it should not be excessive, since the joint presence of various metal cations in the electrolyte can lead to the formation of alloys during the cathode process on the working electrode. In turn, this will affect the appearance of the inversion voltamperogram, which in some cases is an undesirable factor in conducting analytical studies.

In accordance with the above, zinc, gallium and copper were selected for the formation of an electrochemical test system. Cadmium and lead are impractical to include in this kit, since the effect of sulfur-containing organic substances on these metals is much less significant (see table) than zinc, gallium, copper and cobalt. The peak potentials of anodic dissolution of copper and cobalt within the measurement error do not differ from each other. In this case, preference is given to copper, since the values of the currents of its anodic dissolution are significantly higher (about 2 times) than the currents of the anodic dissolution of cobalt.

Following the methodology adopted in this work, at the first stage, measurements were carried out, the results of which were a set of 4000 points, with the help of which the inversion voltamperogram of the electrochemical test system was digitized. At the second stage, when the analyzed organic matter was introduced into the test system, digitization was also performed using a set of 4000 points. The results obtained were normalized to the magnitude of the peak current of the anodic dissolution of copper in the test system, which was assigned a unit value. Next, find the difference between the curves of the test system and the curves obtained after the introduction of organic matter. Such difference dependencies, also consisting of 4000 points, were broken into $N$ the intervals over which the integration was performed in order to present information about each experiment in the form $\mathbf{N}$-dimensional vector. Such a presentation can be considered as a result of multisensory analysis, in which the number of sensors is $N$, since each of the $N$ intervals contains information about the specific effect on the test system of the analyze.

The results of experiments conducted with the above-listed sulfurcontaining organic substances, formed the basis for the formation of their database. When processing the results, the number $N$ is chosen equal to 26 .

Identification of a previously unknown substance is carried out by comparing its vector representation with vector representations of organic substances from a previously collected database. The results of the comparison are displayed on the monitor in the form of a histogram consisting of horizontally 
spaced segments, corresponding to the substances from the database, showing their relative proximity to analyze. The magnitude of these segments is calculated based on the Euclidean distance between the multidimensional vector image of analyze and the vector representations of each organic substance constituting the database. These distances, expressed in relative units, are also displayed on the monitor in the corresponding segments. An example of a histogram obtained by comparing thiobarbituric acid with a database is shown in Fig. 2.

\begin{tabular}{|lll|l|}
\hline 001 & 0,12 & Thiobarbituric acid & \\
\hline 002 & 0,40 & Sulphourea & \\
\hline 003 & 0,50 & Sulfanilamides (Streptocide) & \\
\hline 004 & 0,52 & Sulfosalicylic acid & \\
\hline 005 & 0,62 & Unitiol \\
\hline 006 & 0,84 & Thiamin & \\
\hline
\end{tabular}

Fig. 2. An example of a histogram obtained by comparing thiobarbituric acid with database

In addition to displaying the results of the analysis in the form of a histogram, the developed program allows you to visualize the multidimensional image of each substance in the form of a set of vectors located around the circumference. Such a presentation makes it possible to visually assess the degree of similarity of analyze and any substance embedded in the database. Such a complementary assessment is very useful because in some cases Euclidean distances may be identical for substances of different chemical nature. Examples of visualization of images of various sulfur-containing organic substances are shown in Fig. 3.
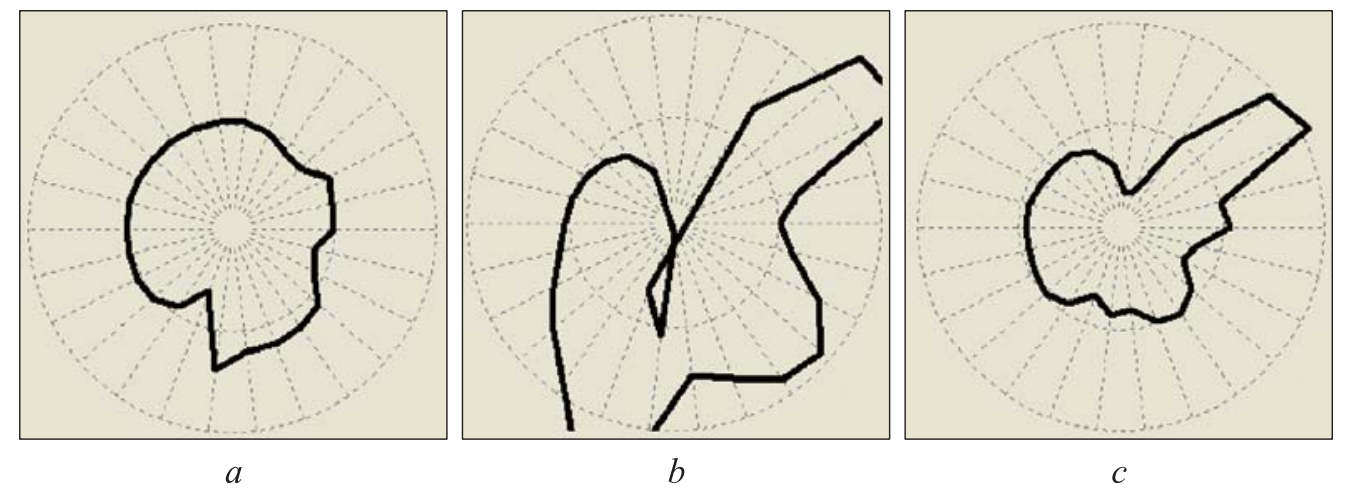

Fig. 3 (part 1). Examples of images of sulfur-containing organic substances: $a$ - sulfosalicylic acid; $b$ - sulfanilamides (streptocide); $c$ - sulphourea 

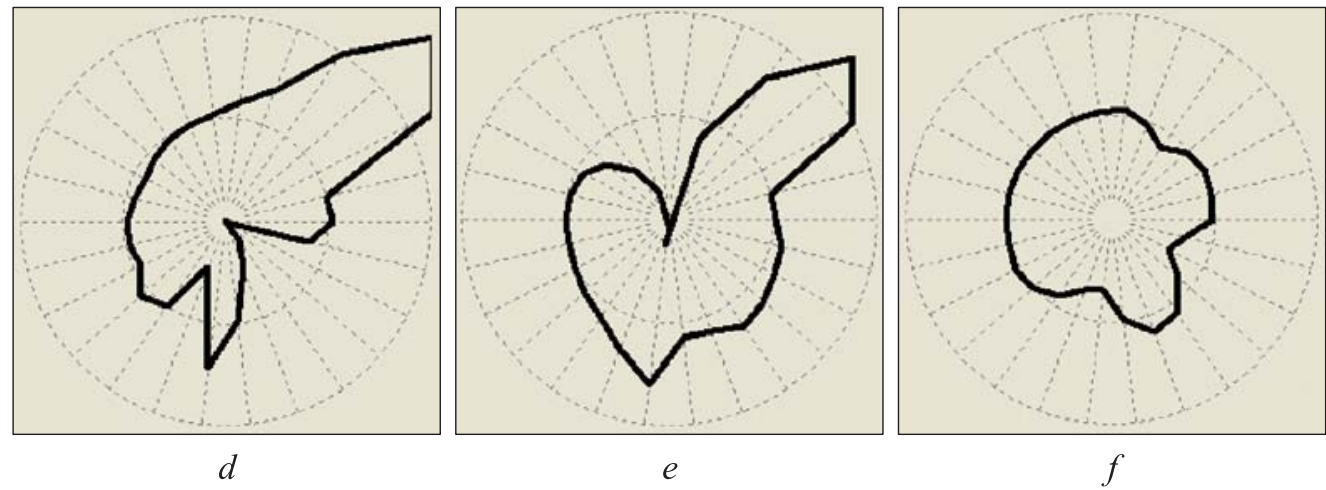

Fig. 3 (part 2). Examples of images of sulfur-containing organic substances: $d$ - unitiol; $e-$ thiobarbituric acid; $f$ - thiamin

Conclusion. The authors showed the necessary to search for a set of metals, based on which an electrochemical test system is created for the detection and identification of organic compounds belonging to different classes, is shown. In the paper points out that the authors developed a test system that allows to obtain reliable results of electroanalytical studies of sulfur-containing organic substances. This is confirmed by a comparative assessment of the Euclidean distances between the vector representations of the analyzed substances and sulfurcontaining organic compounds that make up the preset database.

The authors developed software for experimental studies, processing of their results, visualization of these results and comparative analysis with the database.

Translated by K. Ivanova

\section{REFERENCES}

[1] Lukovtsev V.P., Doronin A.N., Lukovtseva N.V., et al. Identification of alkaloids using the stripping voltammetry method. Russ. J. Electrochem., 2009, vol. 45, iss. 7, pp. 810-812. DOI: $10.1134 /$ S1023193509070167

[2] Andreev V.N., Ganshin V.M., Doronin A.N., et al. Sposob elektrokhimicheskogo mul'tisensornogo obnaruzheniya i identifikatsii alkaloidov [Method of electrochemical multisensory alkaloid detection and identification]. Patent 2375705 RF. Appl. 22.08.2008, publ. 10.12.2009 (in Russ.).

[3] Doronin A.N., Tikhonova S.V., Semenova V.A., et al. The effect of papaverine on cadmium electrodeposition on glassy carbon electrode modified with mercury. Russ. J. Electrochem., 2012, vol. 48, iss. 9, pp. 941-943. DOI: 10.1134/S1023193512080034

[4] Kolisnichenko I.I., Klyuev A.L., Ganshin V.M., et al. Express screening of biological objects using multisensory inversion voltammetry with pattern recognition. Prot. Met. Phys. Chem. Surf., 2014, vol. 50, iss. 4, pp. 543-547. DOI: 10.1134/S2070205114040078 
[5] Ganshin V.M., Doronin A.N., Lukovtsev V.P., et al. Multisensory electrochemical analyzer of toxic agents. Vestn. NTC VPK RF, 2016, vol. 9, no. 4, pp. 73-80 (in Russ.).

[6] Lukovtseva N.V., Ganshin V.M., Doronin A.N., et al. Electrochemical sensor for integral determination of toxic substances in "electronic nose" format at monitoring mode. Vestn. Mosk. Gos. Tekh. Univ. im. N.E. Baumana, Estestv. Nauki [Herald of the Bauman Moscow State Tech. Univ., Nat. Sci.], 2017, no. 4, pp. 100-108 (in Russ.).

DOI: $10.18698 / 1812-3368-2017-4-100-108$

[7] Lukovzev V.P., Bobov K.N., Dribinskii A.V., et al. Portable programmed multifunctional research device. Praktika protivokorrozionnoy zashchity [Practice Corrosion Protection], 1999, no. 3 (13), pp. 61-62 (in Russ.).

Lukovtsev V.P. - Cand. Sc. (Eng.), Leading Researcher, A.N. Frumkin Institute of Physical Chemistry and Electrochemistry, Russian Academy of Sciences (Leninskiy prospekt 31, str. 4, Moscow, 119071 Russian Federation).

Lukovtseva N.V. - Researcher, A.N. Frumkin Institute of Physical Chemistry and Electrochemistry, Russian Academy of Sciences (Leninskiy prospekt 31, str. 4, Moscow, 119071 Russian Federation).

Semenova V.A. - Researcher, A.N. Frumkin Institute of Physical Chemistry and Electrochemistry, Russian Academy of Sciences (Leninskiy prospekt 31, str. 4, Moscow, 119071 Russian Federation).

\section{Please cite this article as:}

Lukovtsev V.P., Lukovtseva N.V., Semenova V.A. Electrochemical Test System for Analysis of Sulfur-Containing Organic Substances. Herald of the Bauman Moscow State Technical University, Series Natural Sciences, 2019, no. 1, pp. 88-95.

DOI: 10.18698/1812-3368-2019-1-88-95 